\title{
Research Status of Rolling Friction Isolation Technology
}

Bo Dong ${ }^{1, a}$, "Xiaosheng Song ${ }^{1, b}$, Qinghua $\mathrm{Li}^{1, \mathrm{c}}$ and Han Zhang ${ }^{1, \mathrm{~d}}$

${ }^{1}$ North China University of Science and Technology, Tangshan, Hebei, China

a919842954@qq.com, b13832809318@163.com, ${ }^{\text {c3} 372297037 @ q q . c o m, ~ d 309081853 @ q q . c o m ~}$

Keywords: Base isolation; Rolling friction isolation; Friction coefficient

Abstract. As a seismic method, rolling friction isolation has been born for over a century. Because of the increase of research interest, it has developed rapidly in recent years. Rolling friction isolation system according to the different types of roller can be divided into rod rolling friction isolation system and ball rolling friction isolation system.

\section{Introduction}

The base isolation is to set up an isolation layer between the upper structure and the lower foundation which can effectively reduce the transmission of seismic energy to the upper part, and to reduce the energy of the seismic energy conversion to ensure the safety of the upper structure. The base isolation is mainly divided into laminated rubber isolation system, friction and sliding isolation system, friction isolation system, rolling friction isolation system and mixed isolation system. Rolling friction isolation system is to add a roller between the top and bottom of the isolation bearings, the system is able to have a certain stiffness to ensure that under normal circumstances, the building is not due to wind loads and other factors and exercise. When the earthquake effect to a certain extent, the roller began to roll up, the seismic energy into kinetic energy, hinder the energy transfer to the upper structure, so as to achieve isolation effect. Rolling friction isolation system roller is generally divided into two types of rolling rod and ball.

\section{Coefficient of Friction}

Rolling friction isolation system mainly rely on the friction between the roller and the board to consume energy, the friction is one of the key datas. The friction is proportional to the friction coefficient. Therefore, the friction coefficient at the contact surface between the roller and the bottom plate is one of the key factors affecting the isolation performance of the rolling friction isolation system. Nan Ge, Youpo Su etc., according to the kinetic theory, analyzed the multi-degree of freedom model and isolated effects of a return spring - roller friction pendulum isolation system. Finding that the roller has better reset capacity and isolation effect, when the stiffness coefficient of the return spring is $0.1 \%$ of the stiffness coefficient of the building and the rolling friction coefficient is about $0.01 \sim 0.03$. The isolation efficiency of the isolation system can reach more than $80 \%$ when the stiffness coefficient and the friction coefficient of the spring are selected properly [1].

\section{Ball Rolling Friction Isolation System}

The ball rolling friction isolation system, because of the characteristics of the ball, needs to set the structure with limit function, a curved groove can limit and can provide for gravity reset function, so it is adopted by many researchers. The selection of the groove section type has great influence on the isolation performance of the system.

Base Groove Section. R.S. Jangid studied the base of the groove section of the parabolic roller rolling friction isolation bearing, finding that the restoring force of the ball is proportional to the displacement; in the case of uniaxial, it is modeled as a linear model. The isolated bearing has a constant isolation frequency and has proved that this feature is not conducive to the isolation performance of the bearing [2]. 
Z.A. Kemeny studied a ball rolling friction bearing with a tapered groove base and found that the restoring force was almost constant when the platform rotation was small; the acceleration of the ball was limited by the inclination of the tapered groove [3].

P. Scott, Harvey Jr. and Henri P. Gavin, based on the idea of the above two scholars, designed a ball rolling friction bearing with a base that near the center of the groove for a parabolic shape and the outer ring is a conical, as shown in Fig.1. There is an inflection point between the parabolic area and the tapered area. The large amplitude free response of the isolation system is very sensitive to the initial conditions. Under strong earthquakes, the support can limit the acceleration and displacement of the superstructure to a low level [4].

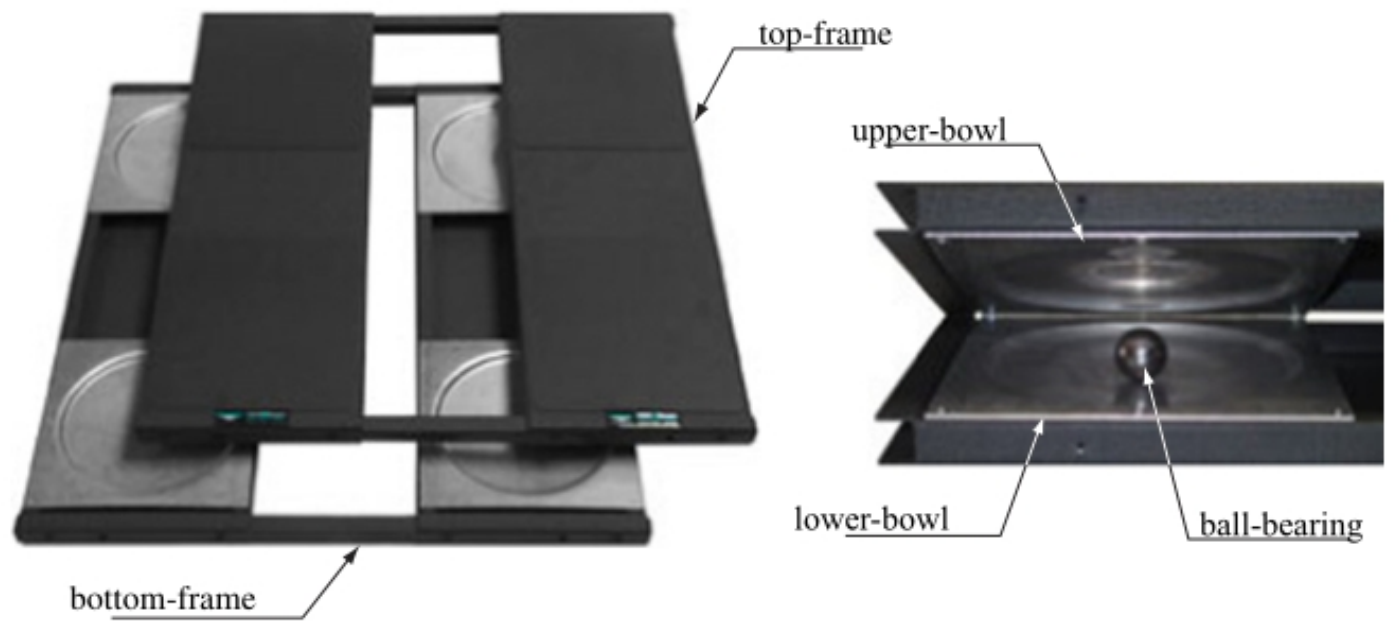

Fig. 1 The ball rolling friction bearing designed by P. Scott etc..
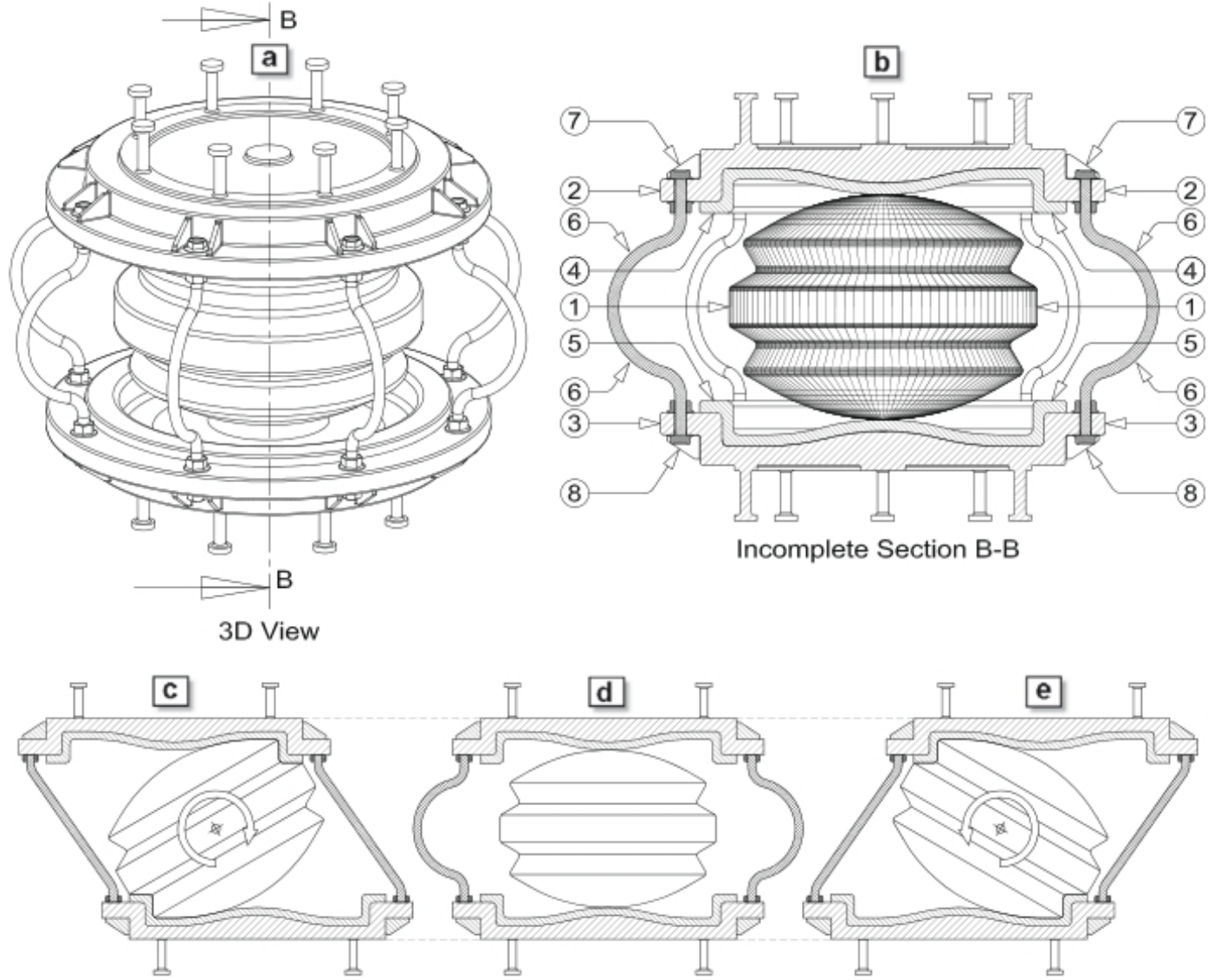

Fig. 2 The roll-n-cage rolling friction bearing designed by M. Ismail etc..

The Shape of Rolling Ball. The ball is one of the key components of this kind of rolling friction isolation bearing, and its appearance shape also affects the isolation performance and stability of the isolation system. The shape of the ball is mostly spherical, P. Scott, Harvey Jr. and Henri P. Gavin 
proposed two opposing intaglio plates that place spherical roller bearings, which can be reset by gravity, but it's lack of the damping device and the pull-out device [4].

M. Ismail, J. Rodellar, and F. Ikhouane proposed a roll-n-cage rolling friction bearing with a roller of alien balls. The alien ball will limit the reciprocating motion within this displacement after scrolling to the predetermined displacement value, as shown in Fig. $2 \mathrm{c}-\mathrm{e}$, this bearing has a gravity reset function. The Part 6 in Fig. 2 is the metal yield dampers with the functions of buffing and consuming energy [5].

\section{Rod Rolling Friction Isolation System}

Rod rolling friction isolation system is set tosome rods between the upper and lower floor, when the earthquake came, multi-dimensional earthquake will make the rods rolling according to their fixed direction. The rolling direction of rod is fixed and it can only move in one direction, so the rods are normally set up at least in two orthogonal directions.

The Shape of Rolling Rod. The circular section of the roller does not have a self-resetting function, so some researchers consider replacing the circular section with an oval cross section. Jangid RS and Londhe YB proposed the use of elliptical rollers, selected different eccentricities of 0,0.25,0.5 and 0.75 to study, the study showed that the eccentricity closer to the same, the smaller the displacement, the absolute acceleration did not change significantly [6].

Butterworth JW found that non-circular rods such as elliptical rollers or non-concentric rods may cause vertical acceleration of the upper structure, but this vertical acceleration is small enough (10-20\% peak relative to horizontal acceleration) and does not affect the upper structure significantly [7].

Nan Ge, Youpo Su etc. studied the seismic isolation system of eccentric circular roller, according to the theory of multi - body dynamics, set up the calculation model of the anti-segregation system of the circular chute eccentric roller friction and analysised its isolation effect, finding that after setting up the system, the interlayer displacement of the structural seismic response can be drastically reduced. If select the eccentricity of the roller section, the radius of the circular chute and the rolling friction coefficient properly, the isolation effect can be more than $90 \%$, the speed and acceleration of the floor can also be greatly reduced [8].

\section{Prospects}

At present, in the application of isolated system, the laminated rubber bearings is most commonly used, friction sliding isolation system also has lots of applications, rolling friction isolation system is rarely used. But the rolling friction form has a smaller friction coefficient in the form of sliding friction. In this respect, the rolling friction isolation system needs to be further studied, so that it should be used in the field of building isolation.

\section{References}

[1] Nan Ge, Youpo Su and Xingguo Wang: Journal of Hebei Polytechnic University (Natural Science Edition), in Chinese, 2008, 30 (4): 132-136.

[2] R.S. Jangid: Optimum friction pendulum system for near-fault motions, Engineering Structures 27 (2005) 249-259.

[3] Z.A. Kemeny: Ball-in-Cone Rolling Isolation Systems, U.S. Patent 5599106.

[4] P. Scott Harvey Jr., Henri P. Gavin: The nonholonomic and chaotic nature of a rolling isolation system, Journal of Sound and Vibration, 332 (2013) 3535-3551.

[5] M. Ismail, J. Rodellar, F. Ikhouane: Performance of structure-equipment systems with a novel roll-n-cage isolation bearing, Computers and Structures 87 (2009) 1631-1646. 
[6] Jangid RS, Londhe YB: Effectiveness of elliptical rolling rods for base isolation. J Struct Eng ASCE 1998;124:469-72.

[7] Butterworth JW: Seismic response of a non-concentric rolling isolator system. Adv Struct Eng 2006;9:39-53.

[8] Nan Ge, Youpo Su and Xingguo Wang, Xiyuan Zhou: Journal of Wuhan University of Technology In Chinese, 2010,32 (9): 246-251. 